\title{
Time variation of the water maser in ON2 ${ }^{\star}$
}

\author{
E. E. Lekht ${ }^{1,2}$, M. A. Trinidad ${ }^{1,3}$, J. E. Mendoza-Torres ${ }^{1}$, G. M. Rudnitskij ${ }^{2}$, and A. M. Tolmachev ${ }^{4}$ \\ 1 Instituto Nacional de Astrofísica, Óptica y Electrónica, Luis Enrique Erro No. 1, Apdo Postal 51 y 216, 72840 Tonantzintla, Puebla, \\ México \\ e-mail: lekht@inaoep.mx \\ 2 Sternberg Astronomical Institute, 13 Universitetskij prospekt, Moscow, 119992, Russia \\ e-mail: lekht@sai.msu.ru \\ 3 Departamento de Astronomia, Universidad de Guanajuato, México \\ 4 Pushchino Radio Astronomy Observatory, Astrospace Center of the Lebedev Institute of Physics, Russian Academy of Sciences, \\ Pushchino, Moscow Region, 142290, Russia \\ e-mail: tolm@prao.psn.ru
}

Received 23 May 2005 / Accepted 26 April 2006

\begin{abstract}
Context. The results of monitoring of the water-vapour maser in the 1.35-cm line toward ON2 in 1995-2004 are reported.

Aims. The main goal was to study variations of the $\mathrm{H}_{2} \mathrm{O}$ maser emission on a long (10 years) time interval, in particular, velocity drifts and correlation between fluxes in various spectral features.

Methods. The observations were carried out on the RT-22 radio telescope of Pushchino Radio Astronomy Observatory.

Results. The emission of the northern component (ON2 N) took place in a broad interval of radial velocities, from -12 to $9 \mathrm{~km} \mathrm{~s}^{-1}$. The total $\mathrm{H}_{2} \mathrm{O}$ emission has two variability components: slow and flaring. The period of the former component is most likely between 25 and 30 years. The flaring component has a cyclic character with a period from 1.1 to 2.6 years. The alternation of the activity cycles was accompanied by changes in the velocity structure of the $\mathrm{H}_{2} \mathrm{O}$ spectra. There is good correlation between the variations in the integrated flux and velocity centroid, as well as between the emissions in various segments of the $\mathrm{H}_{2} \mathrm{O}$ spectrum. We suggest that long-term variations and oscillations of the water-maser emission can be related to the non-stationarity and anisotropy of bipolar molecular outflow from the B-type star in the ultracompact H II region.
\end{abstract}

Key words. masers - lines: profiles

\section{Introduction}

The source ON2 is located in the intricate Cygnus $\mathrm{X}$ complex at a distance of $5.5 \mathrm{kpc}$ (Wink et al. 1982). The northern molecular cloud, also called $\mathrm{ON} 2 \mathrm{~N}$ and $\mathrm{ON} 2 \mathrm{~S}$, contains an ultracompact H II region with a cometary morphology. Shepherd \& Churchwell (1996) found a bipolar molecular outflow near the UC H II region G75.78+0.34 from ${ }^{12} \mathrm{CO}$ line observations and calculated that its source can be an early-type (B) star on the basis of the parameters of this outflow. The UC H II region produces a bow shock, which results from the relative motion of the B-type star and the surrounding dense molecular gas (Wood \& Churchwell 1989). The strong masers in the lines of $\mathrm{OH}$ (Elldér et al. 1969) and $\mathrm{H}_{2} \mathrm{O}$ (Forster et al. 1978) are closely associated with the UC H II region.

The water-vapour maser was detected toward ON2 by Johnston et al. (1973). Genzel \& Downes (1977) have shown that the emission comes from two spatially isolated regions ( $N$ and $\mathrm{S}$ components). The angular separation between the components is $\approx 80^{\prime \prime}$. Subsequent VLBI observations by Hofner \& Churchwell (1996) have detected three groups of maser spots in $\mathrm{G} 75.78+0.34$. The emission of the main group ( $\mathrm{N}$ component) is in the velocity interval $-3<V_{\mathrm{LSR}}<15 \mathrm{~km} \mathrm{~s}^{-1}$. The group has an angular size of about $1^{\prime \prime} \times 0.4^{\prime \prime}$ and is located near the bow shock. It is associated with a hot $(T>100 \mathrm{~K})$, dense

* Figure 10 is only available in electronic form at http://www. edpsciences.org
$\left(n\left(\mathrm{H}_{2}\right) \approx 10^{7} \mathrm{~cm}^{-3}\right)$ molecular clump in the close vicinity of the UC H II region. Two other groups with the emission at negative velocities are sufficiently compact; they virtually coincide with the position of the S component, as detected earlier by Genzel \& Downes (1977).

The variability of the water maser in ON2 was studied by White \& Macdonald (1979). Two years of their observations have shown wide flux variations in the entire radial velocity range; i.e., the fluxes of both components ( $\mathrm{N}$ and $\mathrm{S}$ ) were subject to considerable changes. A long-term monitoring of the $\mathrm{H}_{2} \mathrm{O}$ maser ON2 was carried out in 1981-1995 (Lekht et al. 1996; Márquez et al. 1998). According to Lekht et al. (1996), the $\mathrm{H}_{2} \mathrm{O}$ maser in ON2 N can have long-term variations of the integrated flux with a period longer than 20 years, as well as faster variations (oscillations) on a timescale of 3-4 years.

After 1995 we continued our monitoring of the water maser in the source ON2; the observations were carried out toward the $\mathrm{N}$ and $\mathrm{S}$ components. In addition, in our analysis of the variations in the integrated flux and velocity centroid we used all the results of the monitoring.

\section{Observations}

The observations of the $\mathrm{H}_{2} \mathrm{O}$ maser toward ON2 reported in this paper were carried out on the 22-m RT-22 radio telescope of Pushchino Radio Astronomy Observatory in 1995-2004. In most cases we observed both 


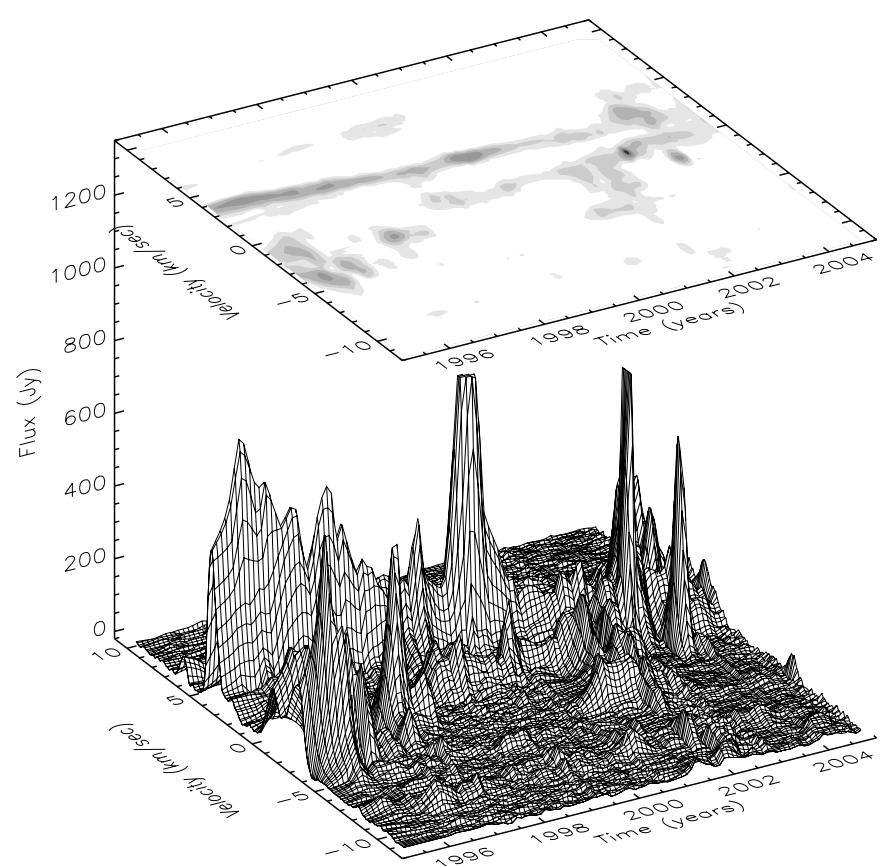

Fig. 1. Three-dimensional presentation of the catalogue of the $\mathrm{H}_{2} \mathrm{O}$ maser emission spectra toward ON2 N for 1995-2004.

components: $\operatorname{RA}(1950)=20^{\mathrm{h}} 19^{\mathrm{m}} 51^{\mathrm{s}} .8$, Dec $(1950)=37^{\circ} 17^{\prime}$ for $\mathrm{N}$ and RA(1950) $=20^{\mathrm{h}} 19^{\mathrm{m}} 49^{\mathrm{s}}$, Dec $(1950)=37^{\circ} 15^{\prime} 58^{\prime \prime}$ for $\mathrm{S}$. For convenience, we will call these sources ON2 N and ON2 S in what follows.

The mean time interval between successive observational sessions was 1.4 months. The system noise temperature was from 100 to $200 \mathrm{~K}$, depending on the weather conditions and source elevation. As far as possible, the observations were carried out at the same position angle to avoid flux variations if the emission of the source was polarized, because the antenna received a single linear polarization. An antenna temperature for an unpolarized pointlike source of $1 \mathrm{~K}$ corresponds to a flux density of $25 \mathrm{Jy}$.

The antenna beamwidth at $22 \mathrm{GHz}$ is $2.6^{\prime}$. When the antenna is pointed toward $\mathrm{ON} 2 \mathrm{~N}$ the emission from the other component $(\mathrm{ON} 2 \mathrm{~S})$ is attenuated by a factor of exactly two, and conversely, when pointed to $\mathrm{ON} 2 \mathrm{~S}$, the emission from $\mathrm{ON} 2 \mathrm{~N}$ is weakened by a factor of two. Since the emission from the southern components comes only at negative velocities, we observed it mainly at $V_{\mathrm{LSR}}<1 \mathrm{~km} \mathrm{~s}^{-1}$. The source ON2 S was occasionally observed in a broader velocity interval. Since the end of 2003 such observations have been carried out on a regular basis.

We also used the method of discrete scanning, i.e., of observations at four points along the line connecting both sources. This allowed us to determine whether the features belong to the sources. These observations were performed in 2003-2004. It should be noted that the emission of the source S was appreciably weaker than that of the source N. Thus the observational techniques we utilized allowed us to separate the emission of the source $\mathrm{S}$ from that of N. This was used in plotting Figs. 2 and 4-9.

The signal was analysed by a 128-channel spectrometer with a resolution of $7.5 \mathrm{kHz}$; at $22 \mathrm{GHz}$ this corresponds to $0.101 \mathrm{~km} \mathrm{~s}^{-1}$. The total analysis bandwidth is about $13 \mathrm{~km} \mathrm{~s}^{-1}$. The required reception bandwidth was provided by retuning the frequency of the first local oscillator. The data were processed at the Instituto Nacional de Astrofísica, Óptica y Electrónica (INAOE), Tonantzintla, Puebla, México.

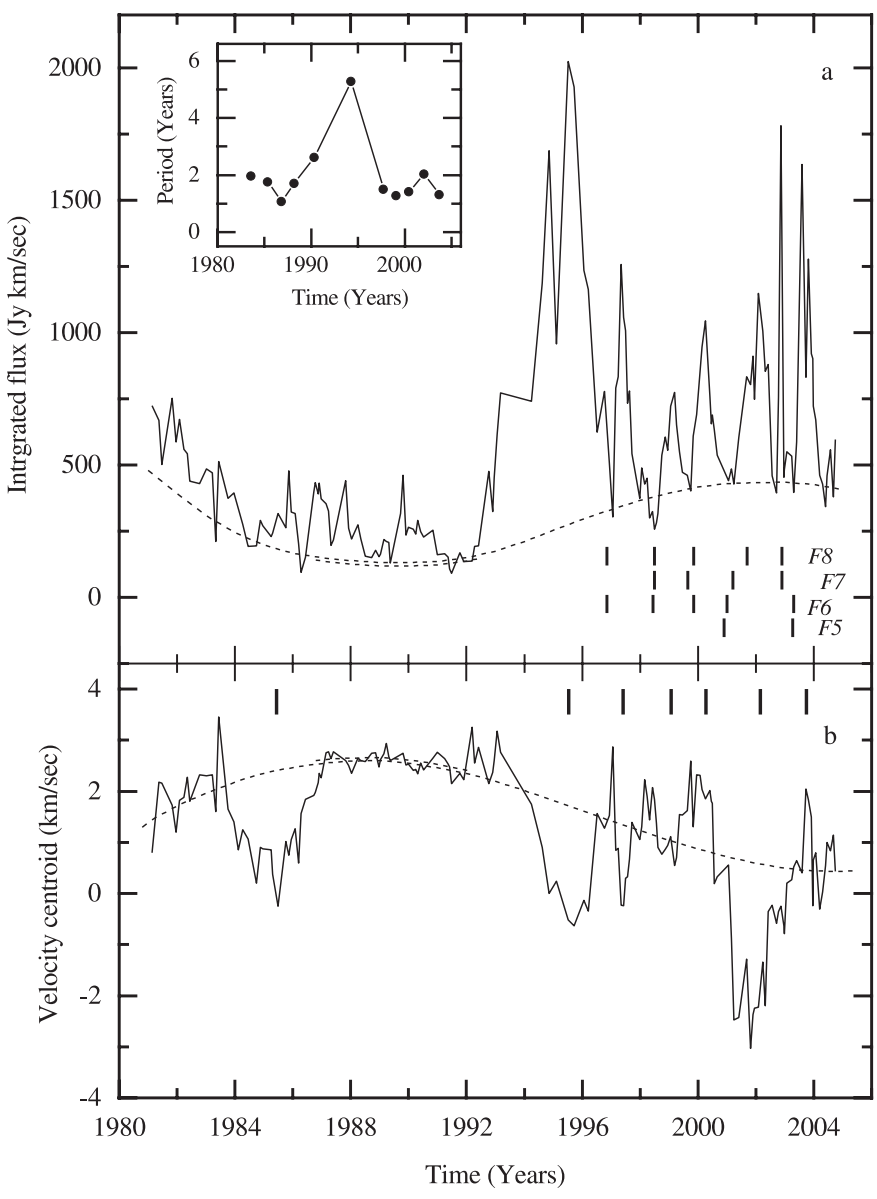

Fig. 2. Variations in the integrated flux a) and velocity centroid b). Dashed lines: smooth curves showing the long-term variability (see text). Vertical bars in the top panel show the emission minima of individual groups of features $F 5-F 8$ according of Figs. 5-8, respectively, and in the bottom panel they denote activity cycles in which $F_{\text {int }}$ and $V_{\mathrm{c}}$ correlated.

\section{Results}

The spectra of the water-vapour maser for 1995-2004 toward the source $\mathrm{ON} 2 \mathrm{~N}$ are presented in the online material section. The vertical arrow shows the scale in Janskys.

A three-dimensional (velocity, time, and flux density) plot of the $\mathrm{H}_{2} \mathrm{O}$ maser spectra toward $\mathrm{ON} 2 \mathrm{~N}$ is shown in Fig. 1. This plot includes all the profiles observed in 1995-2004. To produce a three-dimensional image (3D) it was necessary to have a regular grid in the velocity-time space. The velocity intervals are regular but the time intervals are not, because the time interval between two consecutive observations changes from one case to the next. Therefore, to obtain a regular grid, we had to interpolate. We made an interpolation using the IDL triangulation procedure. Then we determined the fluxes for the new grid by the method of linear interpolation, using the Trigrid IDL procedure. The contours of the fluxes of the $\mathrm{H}_{2} \mathrm{O}$ maser emission are shown in a panel fixed to the 3D plot.

It is clear that the emission at a mean velocity of $3 \mathrm{~km} \mathrm{~s}^{-1}$, which is permanently present in the spectra, has a regular drift. The intervals of 1995-1996 and 2001-2002 are characterised by a high maser activity and extended spectra. Another ridge, less extended in time, was observed since mid-1998 near zero velocities. The emission at other velocities was not as long-lived as described above. It had a flaring nature. 


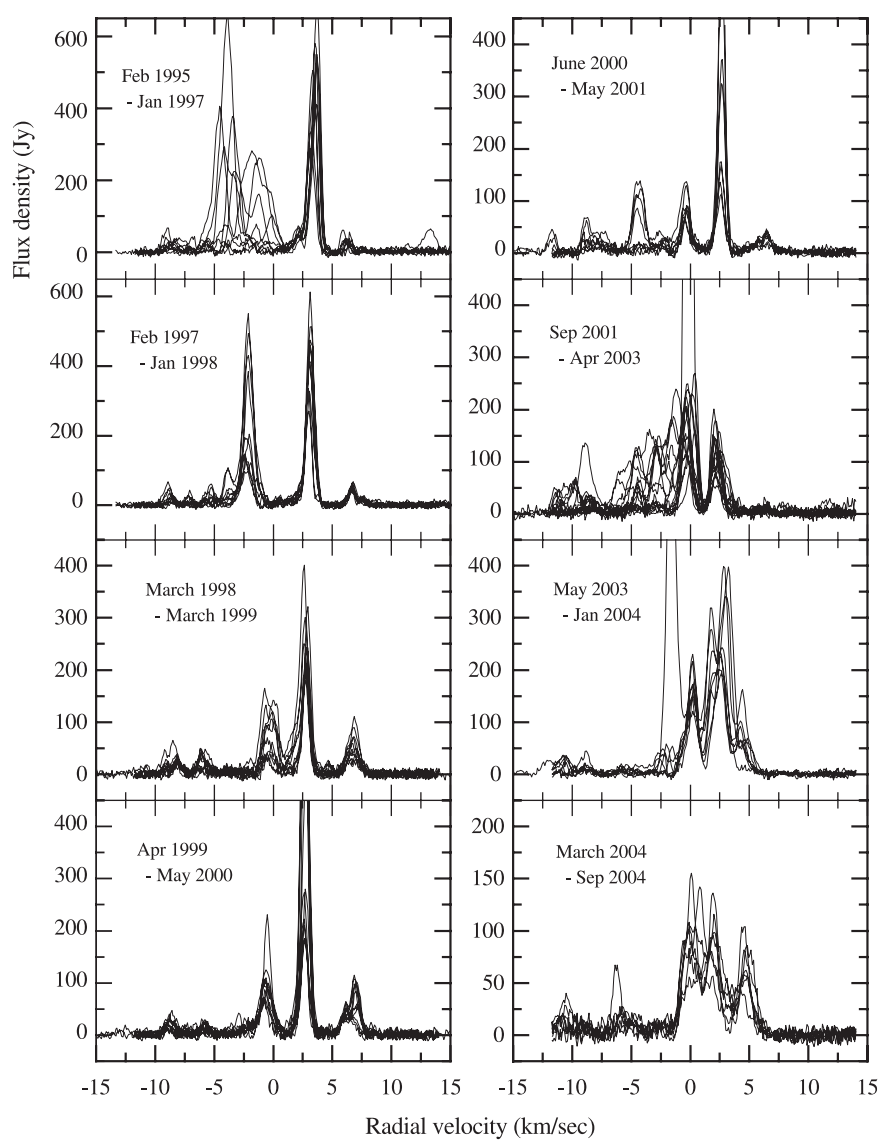

Fig. 3. Superposition of the $\mathrm{H}_{2} \mathrm{O}$ spectra for various time intervals. The interval boundaries are shown.

To study the character of the variability of the integrated flux $\left(F_{\text {int }}\right)$ and velocity centroid $\left(V_{\mathrm{c}}\right)$, we also used the results obtained by Lekht et al. (1996). The variations of these parameters of the $\mathrm{H}_{2} \mathrm{O}$ emission are shown in Fig. 2. In the top panel the fitting was done on the basis of the flux minimum. This enabled us to distinguish two components of the integrated-flux variability. It is clear that the smooth curve of the integrated flux is superposed with faster variations on a timescale of 2-3 years.

We have fitted a third-power polynomial to the velocity centroid (lower panel of Fig. 2). In 1984-1986, 1994.3-1996.6, 1999.4-2000.5, and 2001.1-2002.5, the centroid deviated considerably from its more or less regular variations. These deviations were caused by the appearance or disappearance of the emission from individual groups of features. The polynomial was fitted by the least-square method, but we excluded the points within the above-mentioned time intervals from the complete set. The comparison of the fitted curves shows that the long-term variations of the integrated flux and velocity centroid correlate. Double dashed lines show the interval in which the minimum of $F_{\text {int }}$ coincided with the maximum of $V_{\mathrm{c}}$. In addition, we observe a correlation between the fast variations of these parameters (Fig. 2).

Then we divided the entire period of the monitoring into time intervals. The dividing boundaries were taken at points when the spectrum structure changed considerably. Figure 3 shows the results of this procedure. The limits of the time intervals are indicated. These intervals are close to those of the cyclic variability of the integrated flux (see Fig. 2a). An exception is the period of 1998.4-2001.3, when two maser activity cycles correspond to

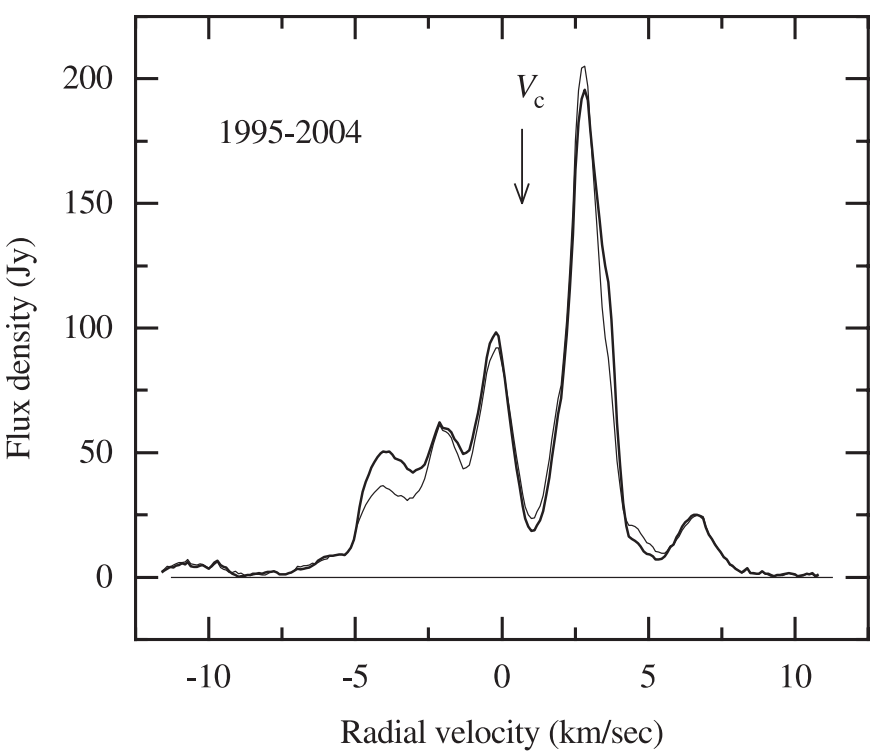

Fig. 4. Average $\mathrm{H}_{2} \mathrm{O}$ spectra obtained by the two methods (see text).

three division intervals. This is connected with strong variations in the $\mathrm{H}_{2} \mathrm{O}$ spectrum structure in 1988-2001.

We calculated the mean spectrum for the time interval of 1995-2004 (Fig. 4). This spectrum was computed as a simple average of all the spectra and with regard to the duration of the intervals. The spectra are almost identical. The arrow shows the position of the velocity centroid for the mean spectrum drawn with the bold curve, $0.7 \mathrm{~km} \mathrm{~s}^{-1}$, which almost coincides with a deep depression at $1.2 \mathrm{~km} \mathrm{~s}^{-1}$.

The flux and velocity variations of the lowest velocity $\mathrm{H}_{2} \mathrm{O}$ emission that we detected toward the northern source are shown in Fig. 5. Figures $6-8$ show the flux variations of individual emission features of $\mathrm{H}_{2} \mathrm{O}$ maser belonging to various spectral groups. Arrows in the lower panels show the activity minima of the groups of the emission features. Maximum probable errors of the observations are shown.

\section{Discussion}

We concentrate here on the variability of the integrated $\mathrm{H}_{2} \mathrm{O}$ flux, velocity centroid, and individual groups of features consisting of spectrally close components.

\subsection{Emission of $O N 2 \mathrm{~N}$ at $V_{L S R}<-5.5 \mathrm{~km} \mathrm{~s}^{-1}$}

The identification of the $\mathrm{H}_{2} \mathrm{O}$ emission features has shown that the main emission toward ON2 comes from the northern source, in the velocity interval $-5.5<V_{\mathrm{LSR}}<9 \mathrm{~km} \mathrm{~s}^{-1}$. Other observations, carried out prior to our monitoring (Cato et al. 1976; White \& Macdonald 1979; Genzel \& Downes 1977), have shown that the maser emission toward ON2 N did not overstep the limits of -5 to $+11 \mathrm{~km} \mathrm{~s}^{-1}$.

Cato et al. (1976) also observed emission at $-6 \mathrm{~km} \mathrm{~s}^{-1}$. However, they supposed that it belonged to another source separated from $\mathrm{ON} 2 \mathrm{~N}$ by $15^{\prime \prime}$. We have drawn attention to the fact that the positions of the features in the two spectra obtained by Cato et al. (1976) at different antenna pointings do not coincide. In addition, it should be noted that the linewidths are large $\left(1.5 \mathrm{~km} \mathrm{~s}^{-1}\right)$. Thus we can conclude that this emission is a superposition of radiation from both sources, $\mathrm{N}$ and $\mathrm{S}$. Precisely this result was obtained in our monitoring. This emission is a 


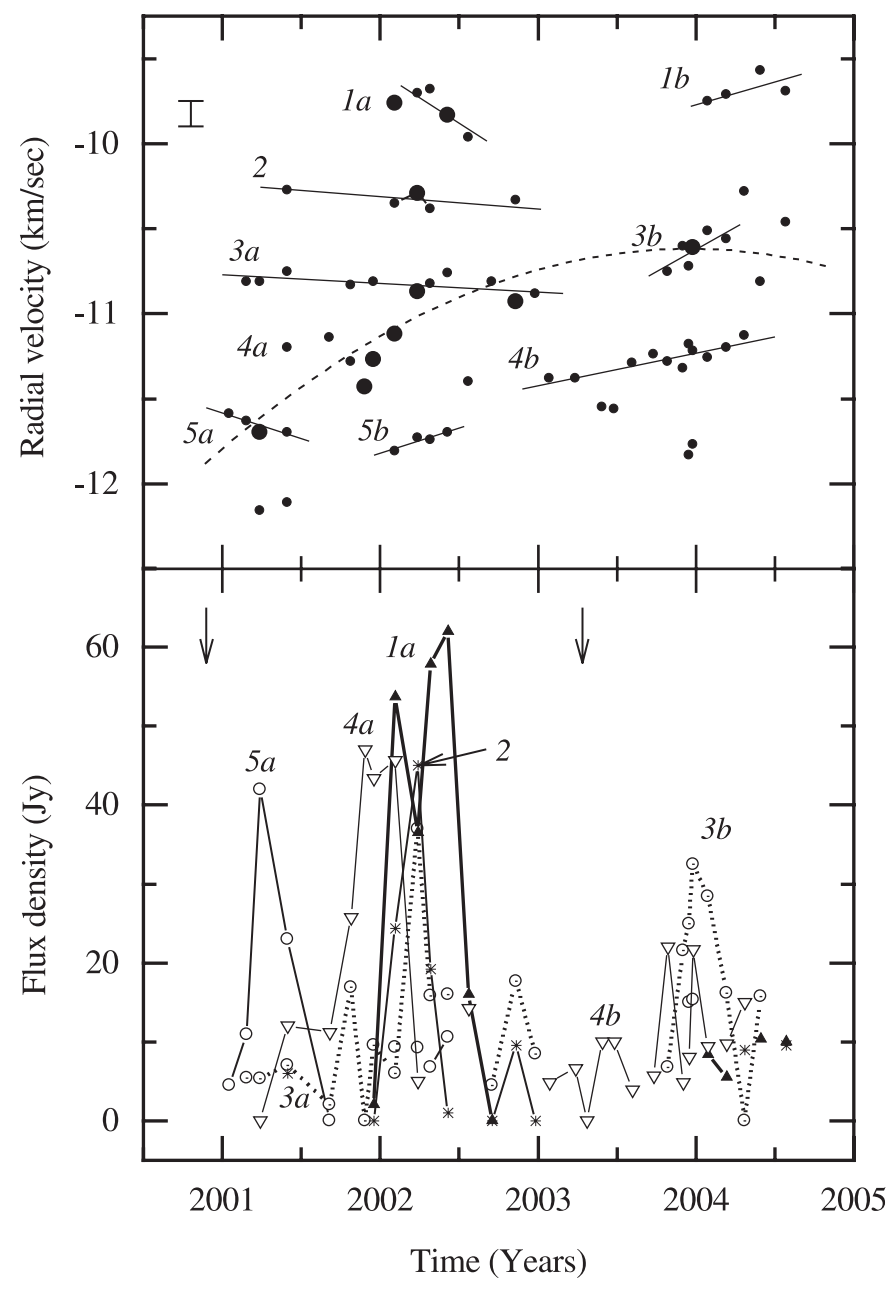

Fig. 5. Flux and radial-velocity variations of the emission features at $V_{\mathrm{LSR}}<-9 \mathrm{~km} \mathrm{~s}^{-1}$. Large circles in the top panel: flux maxima. The positions of the main emission maxima with the exception of features $1 a$ and 2 are approximated with a second-power polynomial (dashed line).

separate group of features in the interval from -7 to $-4 \mathrm{~km} \mathrm{~s}^{-1}$ and is strongly variable. We observed it from both sources. At different epochs, the emission from one of the sources was prevailing. In spite of the strong variability of this emission, down to its disappearance for several years, the feature at a velocity near $-6 \mathrm{~km} \mathrm{~s}^{-1}$ is persistently reproduced 30 years already; this testifies to the existence of stable clumps of material in ON2.

A strong short-lived flare was observed in October 2001 at $-9 \mathrm{~km} \mathrm{~s}^{-1}$ with a flux density of $135 \mathrm{Jy}$. The emission at the extreme negative velocities (below $-10 \mathrm{~km} \mathrm{~s}^{-1}$ ) was recorded for first time by us at the beginning of 2001. This emission represents a compact group of a few features (Fig. 5). The longest lived is the emission feature at a mean velocity of $-11 \mathrm{~km} \mathrm{~s}^{-1}$. Its peak flux density reached $50 \mathrm{Jy}$. The majority of the features display a drift in radial velocity and fast flux variability. The observed consecutive maxima are arranged in the order of increasing radial velocity.

Thus, our long-term monitoring has allowed us to detect the emission toward $\mathrm{ON} 2 \mathrm{~N}$ in the entire range of negative radial velocities down to $-12 \mathrm{~km} \mathrm{~s}^{-1}$ and the existence of stable structures in the source. It is quite probable that the emission we have found forms a separate group, which is absent on the map of Hofner et al. (1996) and was not detected in earlier observations

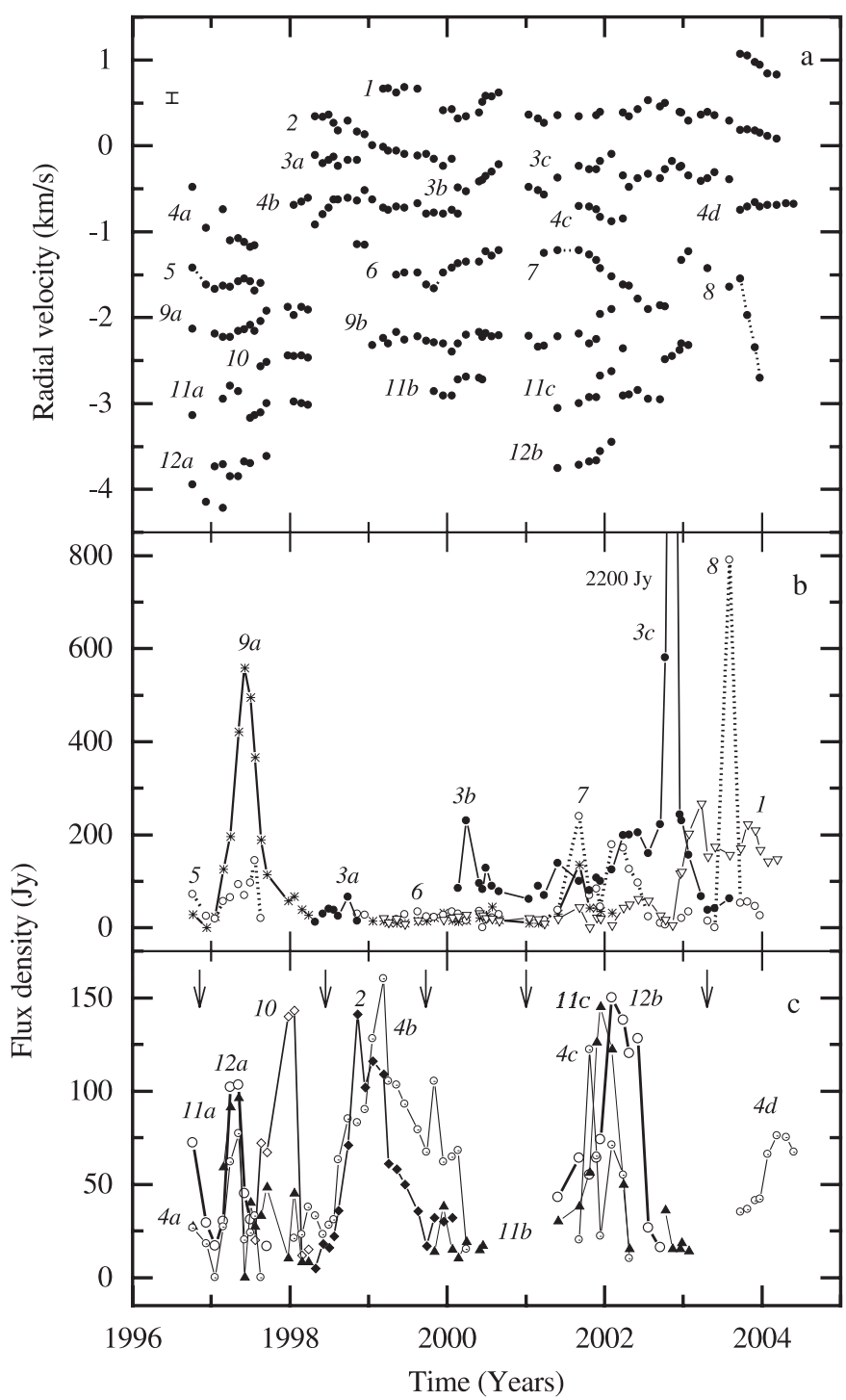

Fig. 6. Flux variability of the emission features at $-5<V_{\mathrm{LSR}}<$ $1 \mathrm{~km} \mathrm{~s}^{-1}$.

in 1977 (Genzel \& Downes 1977) and 1987 (Comoretto et al. 1990).

\subsection{Long-term variations in the $\mathrm{H}_{2} \mathrm{O}$ maser emission}

To study the variability of the integrated flux and velocity centroid, we used the results of our entire monitoring of ON2 for 1981-2004. Thus, the total duration of the monitoring was almost 24 years.

According to Fig. 2, the variability of $F_{\text {int }}$ can be represented as a superposition of slowly varying and flaring components. Using the data obtained prior to our monitoring, Lekht et al. (1996) supposed that the period of the slow variations exceeds 20 years. This estimate was based on the two main maxima of the maser activity (1975 and 1995). Using the monitoring of 19812004 alone, we obtained a smoother curve for the integratedflux variations. The minimum activity falls on the minimum of the smooth curve, while the absolute maximum of the emission in our monitoring (1995-1996) is not at the maximum of the smooth curve. If we determine the period from the smooth curve, it is $\sim 24-25$ years. From two main maxima for the entire time 


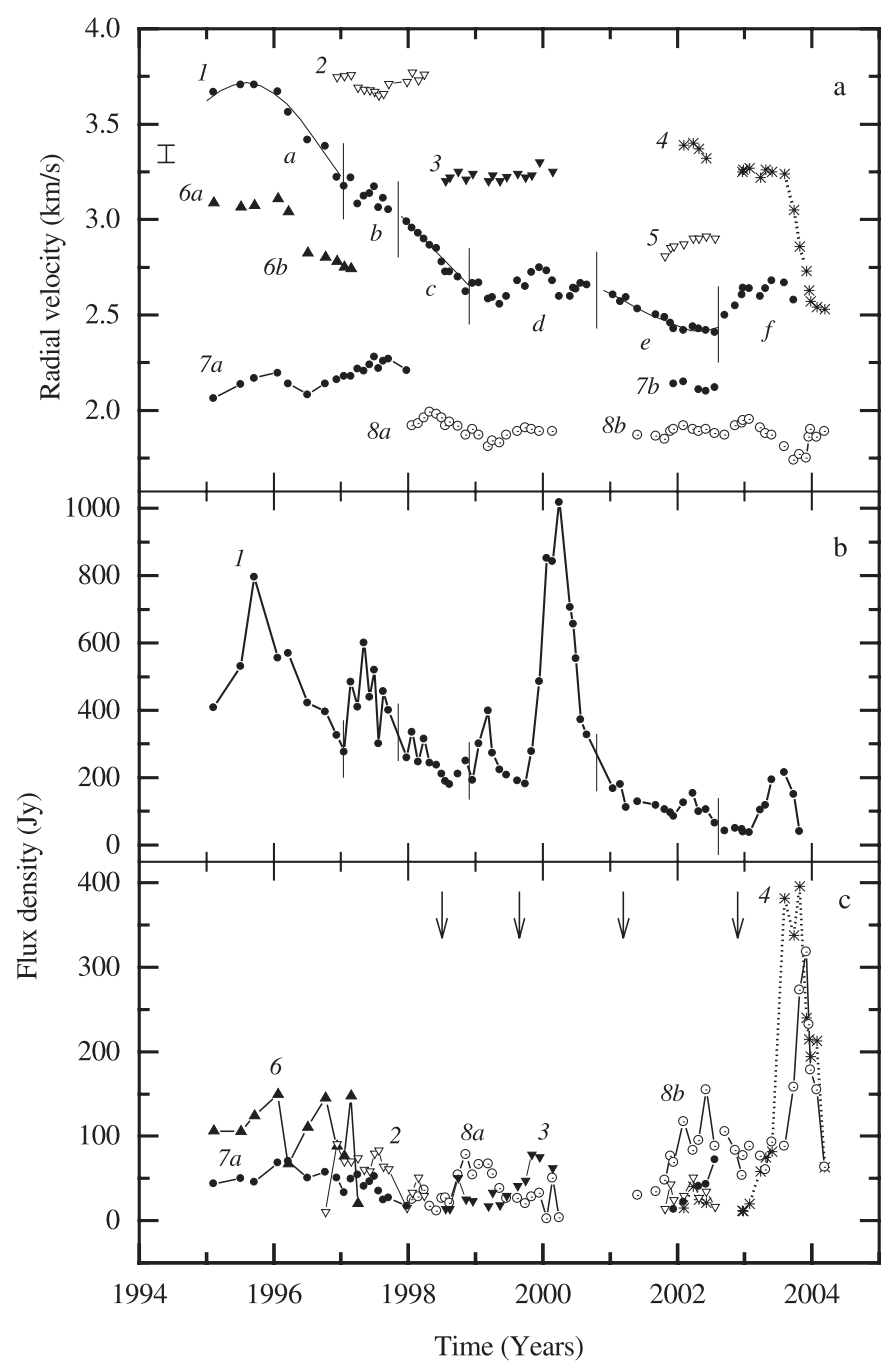

Fig. 7. Flux variability of the emission features of main group (see text).

span of the observations of ON2 (1973-2004), the period can be estimated as $\sim 19-20$ years.

The variability of the velocity centroid (as well as that of the integrated flux) is well approximated by two components: slow drift and strong oscillations. We can assert the existence of a correlation between the two curves with good confidence, reflecting the slow variations in $F_{\text {int }}$ and $V_{\mathrm{c}}$. In 1987-1991 the minimum of $F_{\text {int }}$ corresponded to the maximum of $V_{\mathrm{c}}$ (Fig. 2). The smooth curve of the $V_{\mathrm{c}}$ drift also enabled us to estimate the period $T$ of the long-term variability of the maser source, $T \sim 30$ years. To specify the character of the centroid drift, a longer time series of the observations is required.

\subsection{Flare maser activity}

Fast variations of the integrated flux (oscillations) happened throughout the time interval of 1981-2004. This was especially conspicuous in 1995-2004. In accordance with that we have 10 activity cycles with a duration $(P)$ from 1.1 to 2.6 years and one (the period of the strongest flare) of 5.3 years. The variations in parameter $P$ are not chaotic: there is a fair time dependence (see insert in Fig. 2). The oscillations of the integrated flux and velocity centroid correlated with each other and were caused by the variations in the structure of the spectra themselves - by appearance or disappearance of groups of features (Fig. 3).

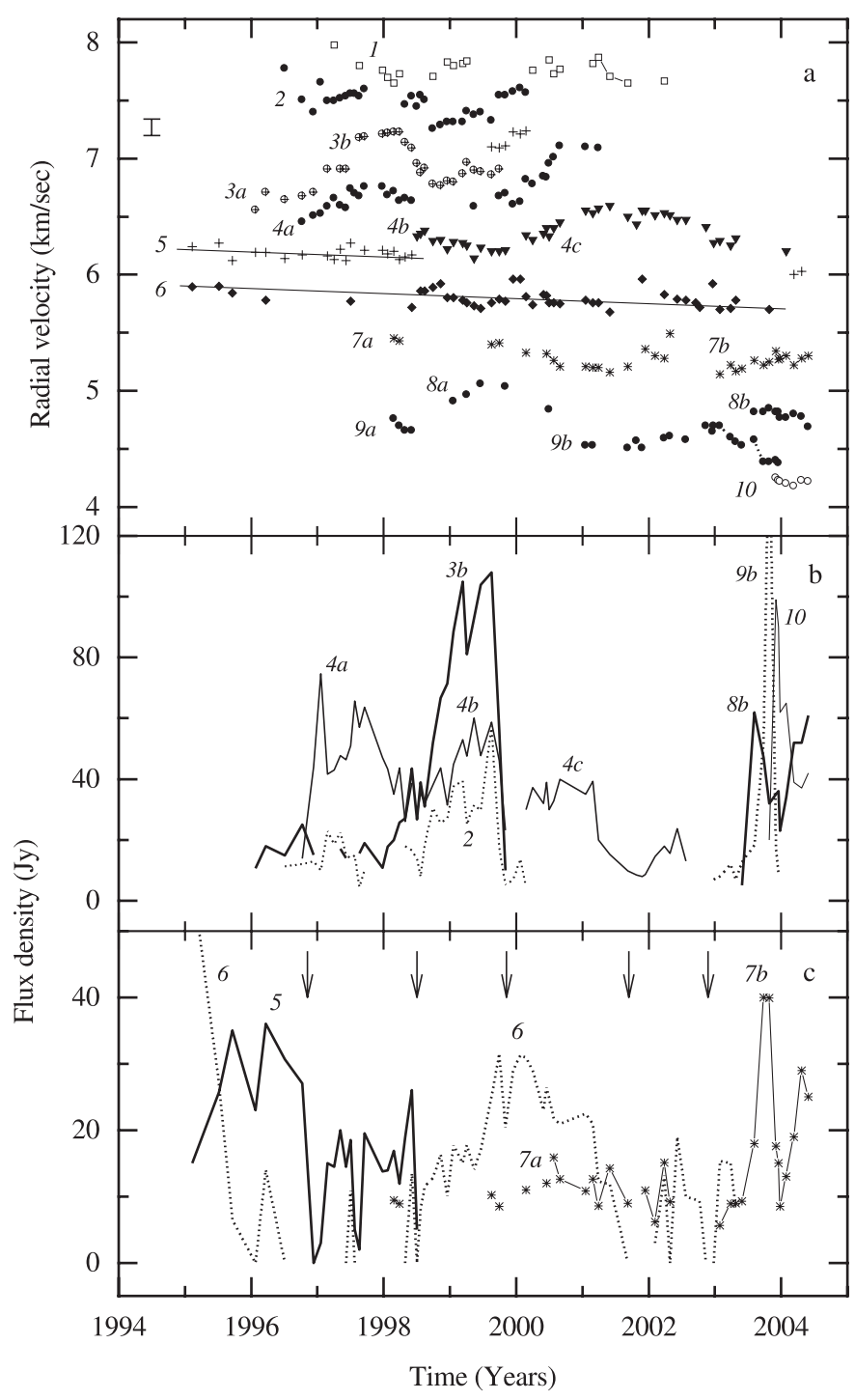

Fig. 8. Flux variability of the emission features at $4<V_{\mathrm{LSR}}<8 \mathrm{~km} \mathrm{~s}^{-1}$.

\subsection{Individual components of the $\mathrm{H}_{2} \mathrm{O}$ maser}

As can be seen from Figs. 3 and 4, the emission features in the $\mathrm{H}_{2} \mathrm{O}$ spectra form separate groups. On this basis, the spectra can be divided into five intervals: $<-9,-9 \rightarrow-5,-5 \rightarrow 1,1 \rightarrow 4$, and $>4 \mathrm{~km} \mathrm{~s}^{-1}$. In each of them we observed flares of individual features on a shorter timescale than the duration of the activity cycles themselves. The life time of some features did not exceed several months. Nearly all emission features had a radialvelocity drift, which was quite diverse, from linear to sinusoidal. We also observed consecutive short-lived flares of up to four features at closeby $V_{\mathrm{LSR}}$, but they were not arranged in the order of increasing or decreasing velocity.

The rather long-lived components (i.e., those in an active phase during 9 years; see Fig. 7) had a large velocity drift from 3.7 to $2.4 \mathrm{~km} \mathrm{~s}^{-1}$. There is a good correlation between the flux and velocity variations. The most complicated velocity variations were associated with flares. During the decay of the emission, the velocity was monotonically decreasing (segments $a, c$, and $e$ in Fig. 7).

All this suggests a complex structure for the maser spot regions, strong fragmentation of the material, and probably the existence of turbulent motions in them. In the framework of this supposition, the maser spots can be formed by projection of 


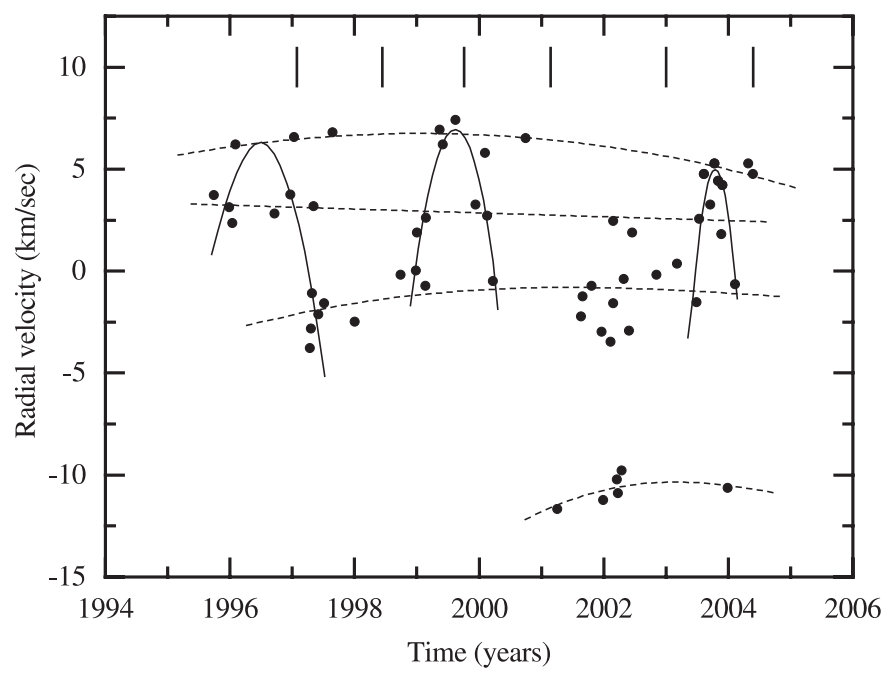

Fig. 9. Positions of the emission maxima of the main features. Dashed lines: fitted positions of the maxima for individual groups of features; solid lines: maxima for all the groups during one activity cycle. Bars show the emission minima of $F_{\mathrm{int}}$.

several clumps of material and can experience important flux and radial-velocity variations. This can be accompanied by spatial displacements of maser spots.

A common feature of all the groups is that the minima of the emission in them virtually coincide in time (see Figs. 2a and 68 ) thereby separating the activity cycles from one another. This correlation suggests that the groups are located at equal distances from the central object and are arranged parallel to the shock front in the UC HII region in the source G75.38+0.34 (Hofner \& Churchwell 1996).

Also visible in Fig. 9 is clustering of the emission features into groups. The scatter from mean values in the maxima positions within each group arises naturally from the dispersion of velocities and positions of the components in the groups.

When the emission at $V_{\mathrm{LSR}}<-9 \mathrm{~km} \mathrm{~s}^{-1}$ was observed, the emission at $V_{\mathrm{LSR}}>4 \mathrm{~km} \mathrm{~s}^{-1}$ was strongly depleted and sometimes absent. This may be due to temporal variations in the bipolar-outflow anisotropy.

\section{Summary}

We have reported the main results of the analysis of the second phase of the $\mathrm{H}_{2} \mathrm{O}$ maser monitoring of the source ON2. The character of the long-term variations and oscillations of the maser emission was derived on the basis of the data of the entire 24-year monitoring.

1. A catalogue (Fig. 10 of the spectra measured toward the source ON2 (N component) during 1995-2004 was compiled (the catalogue is presented in the online material section).

2. We detected the $\mathrm{H}_{2} \mathrm{O}$ maser emission from $\mathrm{ON} 2 \mathrm{~N}$ also in the velocity interval from -12 to $-6 \mathrm{~km} \mathrm{~s}^{-1}$.

3 . The integrated-flux variations can be represented as a superposition of two components: slowly varying and flaring. The period of the former is most likely between 25 and 30 years. The latter component shows a cyclic character of the variations with periods from 1.1 to 2.6 years. An exception is the period of the highest maser activity in ON2, which is estimated as 5.3 years. The alternation of the maser activity cycles was accompanied by structural variations in the $\mathrm{H}_{2} \mathrm{O}$ spectra.

4. Emission features in the $\mathrm{H}_{2} \mathrm{O}$ spectrum form separate groups. Most probably, these groups are associated with individual clusters of maser spots arranged along the bow shock in the immediate vicinity of the cometary UC H II region.

5. The overall variation in the maser may be due to changes in the parameters of the bow shock. In turn, such changes can result from variations in the rate of accretion onto the young stellar object (source of excitation of the UC H II region) and of the bipolar molecular outflow.

Acknowledgements. The 22-m radio telescope is supported by the Ministry of Industry and Science of the Russian Federation (facility registration number 0110). The authors thank the staff of the Pushchino Radio Astronomy Observatory for their extensive help with the observations and also the anonymous referee, whose suggestions have improved the manuscript.

\section{References}

Cato, B. T., Rönnäng, B. O., Rydbeck, O. E. H., et al. 1976, ApJ 208, 87 Comoettto, G., Palagi, F., Cesaroni, R., et al. 1990, A\&AS, 84, 179 Elldér, J., Rönnäng, B., \& Winnberg, A. 1969, Nature 222, 67

Forster, J. R., Welch, W. J., Wright, M. C. H., \& Baudry, A. 1978, ApJ, 221, 137 Genzel, R., \& Downes, D. 1977, A\&A, 30, 145

Hofner, P., \& Churchwell, E. 1996, A\&AS, 120, 283

Johnston, K. J., Sloanaker, R. M., \& Bologna, J. M. 1973, ApJ 182, 67 Lekht, E. E., Márquez, A., \& Mendoza-Torres, J. E. 1996, A\&AS, 120, 415 Márquez, A., Mendoza-Torres, J. E., \& Lekht, E. E. 1998, A\&AS, 128, 1 Shepherd, D. S., \& Churchwell, E. 1996, ApJ, 472, 225 Shepherd, D. S., Churchwell, E., \& Wilner, D. J. 1997, ApJ 482, 355 White, G. J., \& Macdonald, G. H. 1979, MNRAS, 188, 745 Wink, J. E., Altenhoff, W. J., \& Mezger, P. G. 1982, A\&A 108, 227 Wood, D. O. S., \& Churchwell, E. 1989, ApJS, 69, 831 
E. E. Lekht et al.: Time variation of the ON2 water maser, Online Material $p 1$

\section{Online Material}


E. E. Lekht et al.: Time variation of the ON2 water maser, Online Material p 2

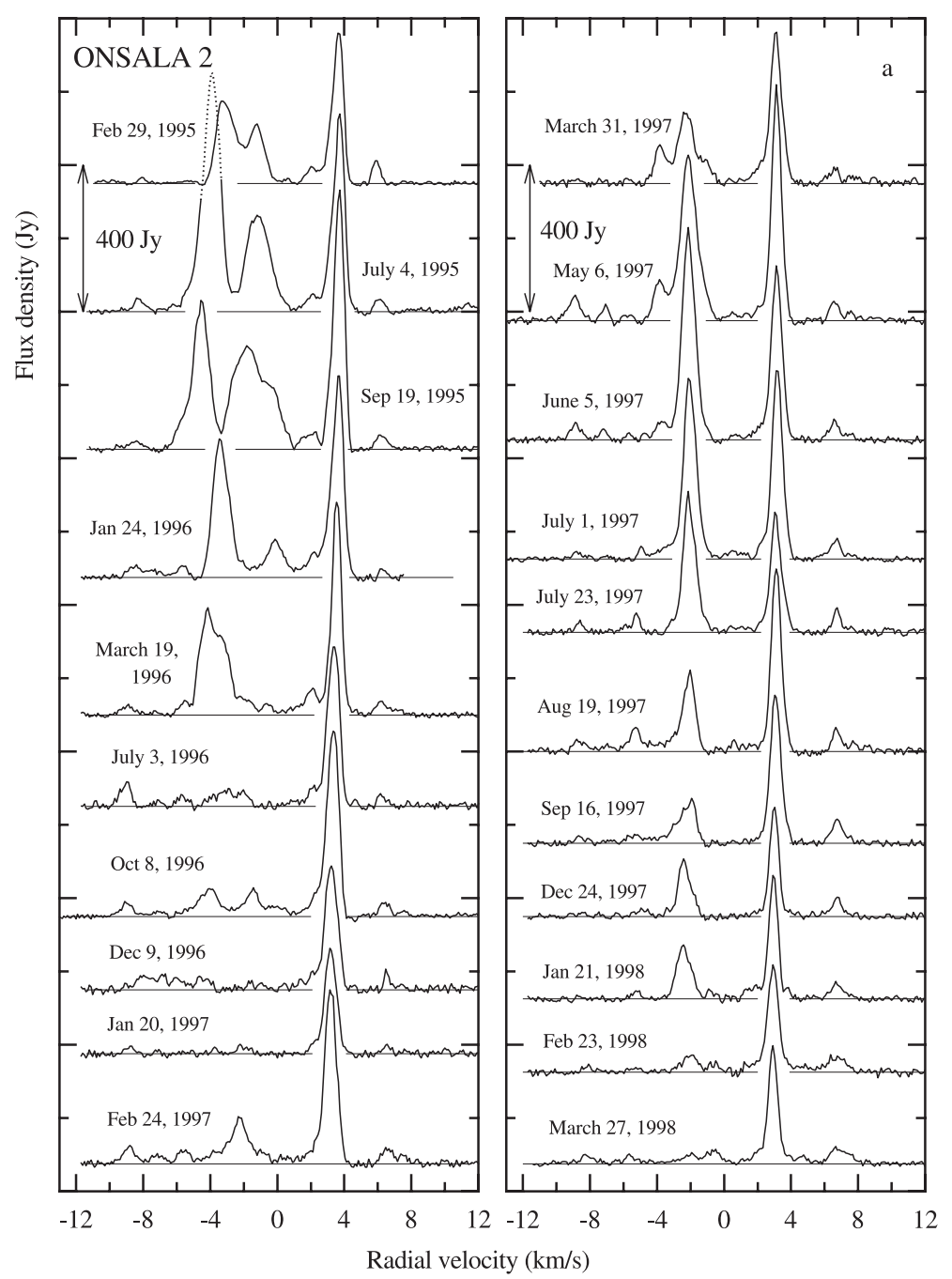

Fig. 10. Spectra of the $\mathrm{H}_{2} \mathrm{O}$ maser emission toward ON2 N for 1995-2004. 
E. E. Lekht et al.: Time variation of the ON2 water maser, Online Material $p 3$

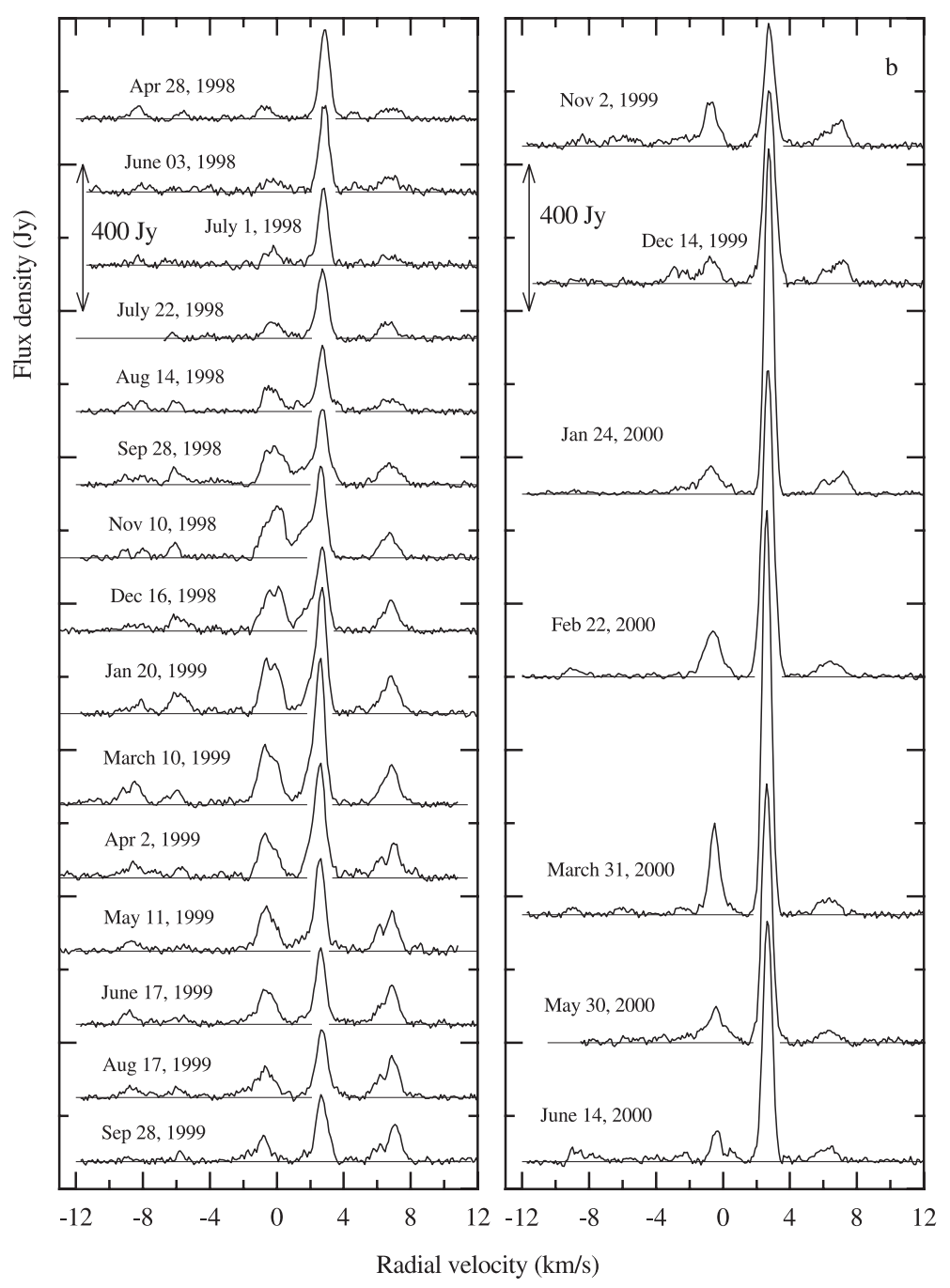

Fig. 10. continued. 
E. E. Lekht et al.: Time variation of the $\mathrm{ON} 2$ water maser, Online Material p 4

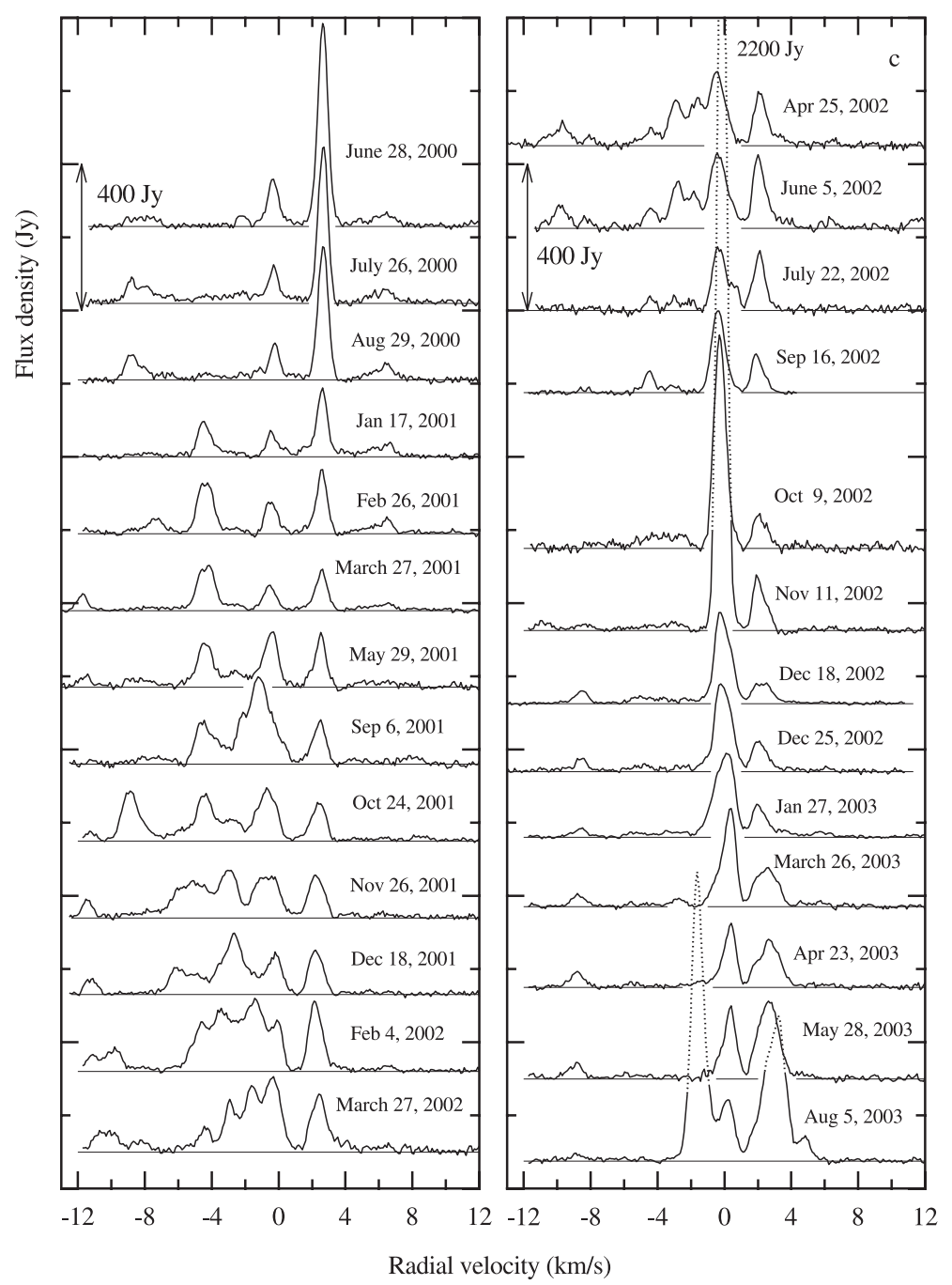

Fig. 10. continued. 
E. E. Lekht et al.: Time variation of the ON2 water maser, Online Material p 5

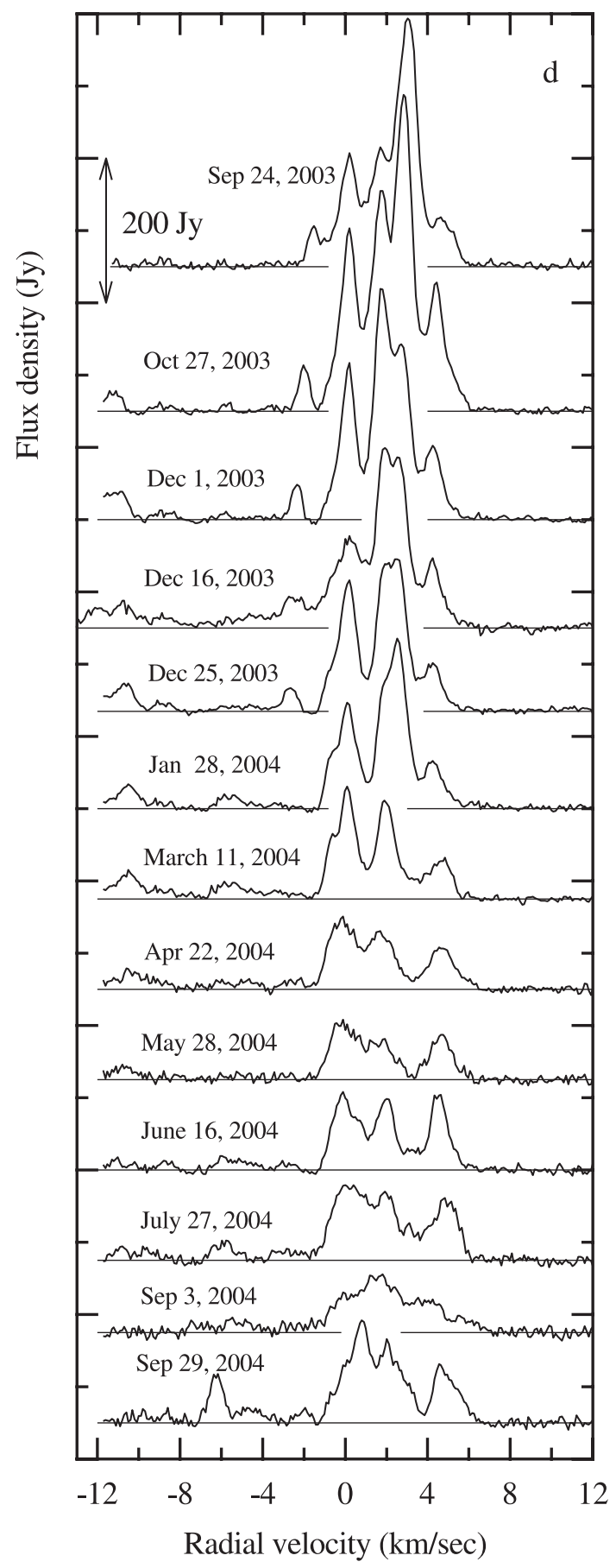

Fig. 10. continued. 